01

\title{
Математическое моделирование процессов тепло- и массопереноса в цилиндрическом канале в зависимости от коэффициента аккомодации тангенциального импульса
}

\author{
(C) О.В. Гермидер, В.Н. Попов
}

Северный (Арктический) фредеральный университет им. М.В. Ломоносова, 163002 Архангельск, Россия

ฯ e-mail: v.popov@narfu.ru

(Поступило в Редакцию 23 декабря 2016 г. В окончательной редакции 11 апреля 1017 г.)

\begin{abstract}
Рассмотрены процессы тепло- и массопереноса в длинном цилиндрическом канале с использованием зеркально-диффузной модели граничного условия Максвелла. В качестве основного уравнения, описывающего кинетику процесса, использовано уравнение Вильямса. В канале поддерживается постоянный градиент температуры. В широком диапазоне изменения числа Кнудсена получены значения потоков тепла и массы через поперечное сечение канала в зависимости от коэффициента аккомодации тангенциального импульса. Построены профили потока тепла. Проведено сравнение с аналогичными результатами, представленными в открытой печати.
\end{abstract}

DOI: 10.21883/JTF.2017.11.45116.2145

\section{Введение}

В связи с развитием направления в динамике разреженного газа, которое связано с разработкой современных технологий MEMS, особое значение приобретают исследования процессов тепло- и массопереноса в микро- и наноканалах в зависимости от модели взаимодействия молекул газа с поверхностью канала [1]. Наиболее известной моделью граничных условий является зеркально-диффузное граничное условие Максвелла. В модели Максвелла функция распределения молекул газа, отраженных от стенок канала, имеет вид [2]

$$
\begin{gathered}
f^{+}\left(\mathbf{r}_{s}^{\prime}, \mathbf{v}\right)=(1-\alpha) f^{-}\left(\mathbf{r}_{s}^{\prime}, \mathbf{v}-2 \mathbf{n}(\mathbf{n v})\right) \\
+\alpha f_{s}\left(\mathbf{r}_{s}^{\prime} \mathbf{v}\right), \quad \mathbf{n v}>0, \\
f_{s}\left(\mathbf{r}_{s}^{\prime}, \mathbf{v}\right)=n\left(z^{\prime}\right)\left(\frac{m}{2 \pi k_{B} T\left(z^{\prime}\right)}\right)^{3 / 2} \exp \left(-\frac{m}{2 k_{B} T\left(z^{\prime}\right)} \mathbf{v}^{2}\right),
\end{gathered}
$$

где $\alpha-$ коэффициент аккомодации тангенциального импульса, $f^{-}\left(\mathbf{r}_{s}^{\prime}, \mathbf{v}-2 \mathbf{n}(\mathbf{n v})\right)-$ функция распределения молекул газа, падающих на стенки, $\mathbf{r}_{s}^{\prime}$ - радиус-вектор точек на стенках канала, $\mathbf{n}$ - вектор нормали к боковой поверхности, направленный в сторону газа, $f_{s}\left(\mathbf{r}_{s}^{\prime}, \mathbf{v}\right)-$ локально равновесная функция распределения. Коэффициент аккомодации тангенциального импульса в модели зеркально-диффузного отражения Максвелла (1) определяет вероятность того, что молекула отразится от стенки диффузно. Таким образом, если $\alpha=1$, то зеркально-диффузное граничное условие переходит в модель диффузного отражения. В случае зеркального отражения коэффициент аккомодации тангенциального импульса имеет нулевое значение.

Другой моделью, описывающей взаимодействие молекул газа с поверхностью, является модель Чер-
чиньяни-Лэмпис (Cercignani-Lampis, CL), в которой учитываются два параметра: коэффициент аккомодации тангенциального импульса $\alpha_{\tau}$ и коэффициент аккомодации кинетической энергии $\alpha_{n}$, связанной с компонентой скорости $\mathbf{v}_{n}$ направленной по нормали к поверхности. B модели CL функция распределения молекул газа, отраженных от поверхности канала, имеет вид [3]

$$
\begin{aligned}
& f^{+}\left(\mathbf{r}_{s}^{\prime}, \mathbf{v}\right)=\frac{1}{\left|\mathbf{v}_{n}\right|} \int_{\mathbf{v}_{n}^{\prime}<0}\left|\mathbf{v}_{n}^{\prime}\right| f^{-}\left(\mathbf{r}_{s}^{\prime}, \mathbf{v}^{\prime}\right) R\left(\mathbf{v}^{\prime} \rightarrow \mathbf{v}\right) d \mathbf{v}^{\prime}, \\
& R\left(\mathbf{v}^{\prime} \rightarrow \mathbf{v}\right)=\frac{m^{2} \mathbf{v}_{n}}{4 \pi^{2} \alpha_{n} \alpha_{\tau}\left(2-\alpha_{\tau}\right)\left(k_{B} T\right)^{2}} \\
& \times \int_{0}^{2 \pi} \exp \left(\frac{\sqrt{1-\alpha_{n}} m \mathbf{v}_{n} \mathbf{v}_{n}^{\prime}}{k_{B} T \alpha_{n}} \cos \phi\right) d \phi \\
& \times \exp \left(\frac{m\left(\mathbf{v}_{n}^{2}+\left(1-\alpha_{n}\right) \mathbf{v}_{n}^{\prime 2}\right)}{2 k_{B} T \alpha_{n}}-\frac{m\left(\mathbf{v}_{\tau}-\left(1-\alpha_{\tau}\right) \mathbf{v}_{\tau}^{\prime}\right)^{2}}{2 k_{B} T \alpha_{\tau}\left(2-\alpha_{\tau}\right)}\right),
\end{aligned}
$$

где $R\left(\mathbf{v}^{\prime} \rightarrow \mathbf{v}\right)$ - ядро рассеяния, $\mathbf{v}^{\prime}, \mathbf{v}-$ векторы скорости падающих и отраженных молекул газа от стенок канала. При $\alpha_{\tau}=1$ и $\alpha_{n}=1$ отражение молекул от поверхности канала является диффузным. В случае $\alpha_{\tau}=0$ и $\alpha_{n}=0$ отражение становится зеркальным.

В рамках модели зеркально-диффузного отражения Максвелла в работах [4-6] исследовалось влияние коэффициента аккомодации тангенциального импульса на тепловой и массовый потоки в канале, образованного двумя бесконечными параллельными пластинами. Для цилиндрического канала результаты вычислений потоков массы и тепла с использованием зеркально-диффузных граничных условий Максвелла и $\mathrm{S}$-модели кинетического уравнения Больцмана приведены в [6], в [7] — на основе БГК. Полученные в [8,9] 
значения коэффициента аккомодации тангенциального импульса показали, что для легких газов, таких как азот, аргон и гелий, коэффициент аккомодации может отличаться от единицы, тогда как для тяжелых газов, например криптона и ксенона, коэффициенты ближе к единице [6]. Для загрязненной поверхности взаимодействие газ-поверхность ближе к диффузному рассеянию, чем взаимодействие в случае специально обработанной гладкой поверхности. Химическая чистка поверхности увеличивает отклонение коэффициента аккомодации от единицы. Если же рассматривается взаимодействие молекул достаточно тяжелого газа с гладкой поверхностью, то можно допускать полную аккомодацию молекул газа поверхностью. В частности, диффузная модель отражения была использована в работах [10-12] для получения значений потоков тепла и массы в прямоугольном канале, в [13] в канале треугольного сечения, в [14-17] в цилиндрическом канале, в [18] в канале эллиптического сечения. В [14-16] результаты получены с использованием численного интегрирования S-модели кинетического уравнения Больцмана. Вместе с тем интерес представляет применение точных аналитических методов. Аналитическое решение уравнения Вильямса в задачах о тепловом крипе в прямоугольном и цилиндрическом каналах получено в $[12,17]$ соответственно. Моделирование процесса массопереноса с граничными условиями Черчиньяни-Лэмпис (Cercignani-Lampis, CL) в прямоугольном канале осуществлялось в [19].

В настоящей работе в отличие от [14-17] получено выражение для потоков тепла и массы в цилиндрическом канале в задаче о тепловом крипе при использовании зеркально-диффузной модели отражения молекул поверхностью канала. В широком диапазоне изменения числа Кнудсена найдены значения потоков тепла и массы в зависимости от коэффициента аккомодации тангенциального импульса.

\section{Постановка задачи. Математическая модель}

Рассмотрим цилиндрический канал радиусом $R^{\prime}$. Предположим, что в канале поддерживается постоянный градиент температуры, направленный вдоль его оси $O z^{\prime}$. Для нахождения функции распределения молекул газа по координатам и скоростям воспользуемся уравнением Вильямса [20], которое в цилиндрической системе координат записывается в виде

$$
\begin{gathered}
\mathrm{v}_{\rho} \frac{\partial f}{\partial \rho^{\prime}}+\frac{\mathrm{v}_{\varphi}}{\rho^{\prime}} \frac{\partial f}{\partial \varphi}+\mathrm{v}_{z} \frac{\partial f}{\partial z^{\prime}}+\frac{\mathrm{v}_{\varphi}^{2}}{\rho^{\prime}} \frac{\partial f}{\partial \mathrm{v}_{\rho}} \\
-\frac{\mathrm{v}_{\rho} \mathrm{v}_{\varphi}}{\rho^{\prime}} \frac{\partial f}{\partial \mathrm{v}_{\varphi}}=\frac{\omega}{\gamma l_{g}}\left(f_{*}-f\right), \\
f_{*}=n_{*}\left(\frac{m}{2 \pi k_{B} T_{*}}\right)^{3 / 2} \exp \left(-\frac{m}{2 k_{B} T_{*}}\left(\mathbf{v}-\mathbf{u}_{*}\right)^{2}\right) .
\end{gathered}
$$

$$
f_{*}\left(\mathbf{r}^{\prime}, \mathbf{v}\right)=f_{0}(C)\left(1+\frac{\delta n_{*}}{n_{0}}+2 \mathbf{C} \mathbf{U}_{*}+\left(C^{2}-\frac{3}{2}\right) \frac{\delta T_{*}}{T_{0}}\right) .
$$

Здесь $n_{0}$ - концентрация молекул газа в начале координат.
Здесь $\mathbf{r}^{\prime}=\mathbf{r}^{\prime}\left(\rho^{\prime}, \varphi, z^{\prime}\right), \rho^{\prime}, \varphi, z^{\prime}-$ координаты молекул газа в цилиндрической системе координат в конфигурационном пространстве; $\mathrm{v}_{\rho}, \mathrm{v}_{\varphi}, \mathrm{v}_{z}$ - проекции вектора скорости $\mathbf{v}$ на оси цилиндрической системы координат, $\omega=\left|\mathbf{v}-\mathbf{u}\left(\mathbf{r}^{\prime}\right)\right|, \mathbf{u}\left(\mathbf{r}^{\prime}\right)-$ массовая скорость газа, $l_{g}=\eta_{g} \beta^{-1 / 2} / p$ - средняя длина свободного пробега молекул газа, $p$ и $\eta_{g}$ - давление и коэффициент динамической вязкости газа, $\gamma=5 \sqrt{\pi} / 4, \beta=m /\left(2 k_{B} T_{0}\right)$, $m$ - масса молекулы газа, $k_{B}$ - постоянная Больцмана, $T_{0}$ - температура газа в некоторой точке, принятой в качестве начала координат. Параметры $n_{*}, \mathbf{u}_{*}$ и $T_{*}$ в функции $f_{*}$ выбираем из условия, что модельный интеграл столкновений удовлетворял законам сохранения числа частиц, импульса и энергии [20]:

$$
\int \omega M_{j} f_{*} d^{3} \mathbf{v}=\int \omega M_{j} f d^{3} \mathbf{v}, \quad j=0-4,
$$

где $\quad M_{0}=1, \quad M_{1}=m \mathrm{v}_{x}, \quad M_{2}=m \mathrm{v}_{y}, \quad M_{3}=m \mathrm{v}_{z}, \quad M_{4}=$ $=m \mathrm{v}^{2} / 2$.

В качестве граничного условия на стенках канала используем модель зеркально-диффузного отражения Максвелла (1).

Рассмотрим установившееся движение газа в канале. Предполагаем, что изменение температуры является малым

$$
G_{T}=\frac{1}{T_{0}} \frac{d T}{d z}, \quad\left|G_{T}\right| \ll 1,
$$

где $G_{T}$ - безразмерный градиент температуры, $T_{0}-$ температура в начале координат, $z=z^{\prime} / R^{\prime}$.

Раскладывая в ряд по градиенту температуры $G_{T}$ выражение (2) и ограничиваясь линейными членами в разложении, получим

$$
f_{s}\left(\mathbf{r}_{s}^{\prime}, \mathbf{v}\right)=f_{0}(C)\left[1+G_{T} z\left(C^{2}-\frac{5}{2}\right)\right] .
$$

Здесь $f_{0}(C)=n_{0}(\beta / \pi)^{3 / 2} \exp \left(-C^{2}\right)-$ абсолютный максвеллиан, $\mathbf{C}=\beta^{1 / 2} \mathbf{v}-$ безразмерная скорость молекул газа.

Функцию $f$ линеаризуем относительно локально равновесной функции распределения $f_{s}\left(\mathbf{r}_{s}^{\prime}, \mathbf{v}\right)$. Принимая во внимание равенство (6) и используя $R^{\prime}$ в качестве масштаба длины, получим

$$
\begin{gathered}
f\left(\mathbf{r}^{\prime}, \mathbf{v}\right)=f_{0}(C)\left(1+G_{T} z\left(C^{2}-\frac{5}{2}\right)+h(\rho, \mathbf{C})\right), \\
\rho=\rho^{\prime} / R^{\prime} .
\end{gathered}
$$

Полагая $n_{*}=n_{0}+\delta n_{*}, T_{*}=T_{0}+\delta T_{*}, \mathbf{U}_{*}=\beta^{1 / 2} \mathbf{u}_{*}$, линеаризуем функцию $f_{*}$ :

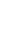


Принимая во внимание, что течение газа по каналу имеет осесимметричный характер, в пространстве скоростей перейдем к сферической системе координат: $C_{p}=C \cos \psi \sin \theta, C_{\varphi}=C \sin \psi \sin \theta, C_{z}=C \cos \theta$. Подставляя выражения (7) и (8) в (3) и учитывая соотношения (4), для нахождения функции $h(\rho, \mathbf{C})$ приходим к уравнению

$$
\begin{gathered}
\left(\left[\cos \psi \frac{\partial h}{\partial \rho}-\frac{\sin \psi}{\rho} \frac{\partial h}{\partial \psi}\right] \sin \theta+G_{T}\left(C^{2}-\frac{5}{2}\right) \cos \theta\right) \gamma K n \\
=\frac{1}{2 \pi} \int C^{\prime} \exp \left(-C^{\prime 2}\right) k\left(\mathbf{C}, \mathbf{C}^{\prime}\right) h\left(\rho, \mathbf{C}^{\prime}\right) d^{3} \mathbf{C}^{\prime}-h(\rho, \mathbf{C}),
\end{gathered}
$$

где $k\left(\mathbf{C}, \mathbf{C}^{\prime}\right)=1+3 \mathbf{C} \mathbf{C}^{\prime} / 2+\left(C^{2}-2\right)\left(C^{\prime 2}-2\right) / 2, K n=l_{g} / R^{\prime}$. Принимая во внимание, что отношение левой и правой частей уравнения (9) равно $\gamma K n$, положим

$$
h(\rho, \mathbf{C})=G_{T}\left(C^{2}-\frac{5}{2}\right) \cos \theta \operatorname{Kn} n Z\left(\rho, \mu_{1}, \mu_{2}\right),
$$

где $\mu_{1}=\cos \psi, \mu_{2}=\sin \theta$.

Подставляя (10) в (9), приходим к уравнению относительно $Z\left(\rho, \mu_{1}, \mu_{2}\right)$ :

$$
\gamma K n\left(\cos \psi \frac{\partial Z}{\partial \rho}-\frac{\partial Z}{\partial \psi} \frac{\sin \psi}{\rho}\right) \sin \theta+Z\left(\rho, \mu_{1}, \mu_{2}\right)+1=0
$$

с граничным условием

$$
Z\left(1, \mu_{1}, \mu_{2}\right)=(1-\alpha) Z\left(1,-\mu_{1}, \mu_{2}\right), \quad \mu_{1}<0 .
$$

Решение уравнения (11) с граничным условием (12) ищем методом характеристик [21]. Система уравнений характеристик для уравнения (11) имеет вид

$$
\frac{d \rho}{\gamma K n \sin \theta \cos \psi}=-\frac{\rho d \psi}{\gamma K n \sin \theta \sin \psi}=-\frac{d Z}{Z+1}=d t .
$$

Интегрируя систему уравнений характеристик (13), находим первые два независимых интеграла:

$$
\rho|\sin \psi|=C_{1}, \quad\left(Z\left(\rho, \mu_{1}, \mu_{2}\right)+1\right) \exp \left(\frac{\rho \mu_{1}}{\gamma K n \mu_{2}}\right)=C_{2} .
$$

Исключая постоянные интегрирования $C_{1}$ и $C_{2}$ из (14) с использованием граничного условия (12), получаем

$$
Z\left(\rho, \mu_{1}, \mu_{2}\right)=W\left(\rho, \mu_{1}, \mu_{2}\right)-1,
$$

$W\left(\rho, \mu_{1}, \mu_{2}\right)=$

$$
\begin{gathered}
\frac{\alpha \exp \left(-w_{1}\left(\rho, \mu_{1}, \mu_{2}\right)\right)}{\exp \left(w_{2}\left(\rho, \mu_{1}, \mu_{2}\right)\right)-(1-\alpha) \exp \left(-w_{2}\left(\rho, \mu_{1}, \mu_{2}\right)\right)} \\
w_{1}\left(\rho, \mu_{1}, \mu_{2}\right)=\frac{\rho \mu_{1}}{\gamma K n \mu_{2}} \\
w_{2}\left(\rho, \mu_{1}, \mu_{2}\right)=\frac{\sqrt{1-\rho^{2}\left(1-\mu_{1}^{2}\right)}}{\gamma K n \mu_{2}}
\end{gathered}
$$

Таким образом, функция распределения $f\left(\mathbf{r}^{\prime}, \mathbf{v}\right)$, определяемая выражением (7), построена. Исходя из статистического смысла функции распределения, находим компоненту вектора потока тепла и массовую скорость газа в канале [2]:

$$
\begin{gathered}
q_{z}^{\prime}\left(\rho^{\prime}\right)=\frac{m}{2} \int\left(\mathrm{v}_{z}-u_{z}\left(\rho^{\prime}\right)\right)\left|\mathbf{v}-\mathbf{u}\left(\rho^{\prime}\right)\right|^{2} \\
\times f\left(\mathbf{r}^{\prime}, \mathbf{v}\right) d^{3} \mathbf{v}=\frac{p_{0}}{\beta^{1 / 2}} q_{z}(\rho), \\
u_{z}\left(\rho^{\prime}\right)=\frac{1}{n\left(z^{\prime}\right)} \int \mathbf{v}_{z} f\left(\mathbf{r}^{\prime}, \mathbf{v}\right) d^{3} \mathbf{v}=\frac{U_{z}(\rho)}{\beta^{1 / 2}},
\end{gathered}
$$

где $q_{z}(\rho), U_{z}(\rho)$ - безразмерные компоненты вектора потока тепла и массовой скорости газа в канале соответственно

$$
\begin{aligned}
& q_{z}(\rho)=\frac{1}{\pi^{3 / 2}} \int \exp \left(-C^{2}\right) C_{z}\left(C^{2}-\frac{5}{2}\right) \\
& \times\left(G_{T} z\left(C^{2}-\frac{5}{2}\right)+h(\rho, \mathbf{C})\right) d^{3} \mathbf{C}=-\frac{3 G_{T} \gamma K n}{2 \sqrt{\pi}} \\
& \times\left(1-\frac{3}{4 \pi} \int_{0}^{\pi} \cos ^{2} \theta \sin \theta d \theta \int_{0}^{2 \pi} W\left(\rho, \mu_{1}, \mu_{2}\right) d \psi\right), \\
& U_{z}(\rho)=\pi^{-3 / 2} \int_{z} \exp \left(-C^{2}\right) \\
& \times\left(G_{T} z\left(C^{2}-\frac{5}{2}\right)+h(\rho, \mathbf{C})\right) d^{3} \mathbf{C}=\frac{G_{T} \gamma K n}{3 \sqrt{\pi}} \\
& \quad \times\left(1-\frac{3}{4 \pi} \int_{0}^{\pi} \cos ^{2} \theta \sin \theta d \theta \int_{0}^{2 \pi} W\left(\rho, \mu_{1}, \mu_{2}\right) d \psi\right) .
\end{aligned}
$$

Приведенные потоки тепла и массы находим согласно [6] как

$$
\begin{gathered}
J_{Q}=\frac{4 \beta^{1 / 2}}{R^{\prime 2} p_{0}} \int_{0}^{R^{\prime}} q_{z}^{\prime}\left(\rho^{\prime}\right) \rho^{\prime} d \rho^{\prime}, \\
J_{M}=\frac{2 m n_{0}}{R^{\prime 2} \beta^{1 / 2} p_{0}} \int_{0}^{R^{\prime}} u_{z}\left(\rho^{\prime}\right) \rho^{\prime} d \rho^{\prime} .
\end{gathered}
$$

Используя выражения (15) и (16) для компоненты вектора потока тепла и массовой скорости газа в канале, получим

$$
\begin{aligned}
& J_{Q}=4 \int_{0}^{1} q_{z}(\rho) \rho d \rho, \\
& J_{M}=4 \int_{0}^{1} U_{z}(\rho) \rho d \rho .
\end{aligned}
$$


Значения $J_{M} / G_{T}$ при различных значениях $\alpha$ и $K n$

\begin{tabular}{c|c|c|c|c|c|c|c}
\hline \multirow{2}{*}{$K n$} & \multicolumn{7}{|c}{$\alpha$} \\
\cline { 2 - 8 } & \multicolumn{3}{|c|}{1} & \multicolumn{2}{|c}{0.8} & \multicolumn{2}{|c}{0.6} \\
\cline { 2 - 8 } & $(19)$ & {$[6]$} & {$[14]$} & $(19)$ & {$[6]$} & $(19)$ & {$[6]$} \\
\hline 0.0001 & 0.0001 & - & - & 0.0001 & - & 0.0001 & - \\
0.0010 & 0.0008 & - & - & 0.0008 & - & 0.0008 & - \\
0.0100 & 0.0083 & - & 0.0116 & 0.0083 & - & 0.0083 & - \\
0.1000 & 0.0765 & 0.1014 & 0.1020 & 0.0779 & 0.0962 & 0.0792 & 0.0908 \\
0.5000 & 0.2705 & 0.3016 & 0.3027 & 0.2968 & 0.3118 & 0.3242 & 0.3230 \\
1.0000 & 0.3881 & 0.3959 & 0.3968 & 0.4537 & 0.4372 & 0.5271 & 0.4865 \\
2.0000 & 0.4977 & 0.4779 & 0.4784 & 0.6221 & 0.5648 & 0.7755 & 0.6769 \\
5.0000 & 0.6080 & 0.5675 & - & 0.8183 & 0.7244 & 1.1087 & 0.9435 \\
10.000 & 0.6632 & 0.6210 & 0.6209 & 0.9288 & 0.8297 & 1.3193 & 1.1341 \\
100.00 & 0.7376 & 0.7243 & 0.7210 & 1.0942 & 1.0530 & 1.6718 & 1.5775 \\
1000.0 & 0.7502 & 0.7486 & 0.7469 & 1.1252 & 1.1166 & 1.7450 & 1.7237 \\
10000 & 0.7520 & - & - & 1.1283 & - & 1.7550 & -
\end{tabular}

Из сравнения выражений (17) и (18) следуют равенства

$$
U_{z}=-\frac{2}{9} q_{z}, \quad J_{M}=-\frac{2}{9} J_{Q} .
$$

Значения приведенного потока массы $J_{M} / G_{T}$ вычислены согласно (19) в зависимости от коэффициента аккомодации тангенциального импульса $\alpha$ методом численного интегрирования Монте-Карло для всего диапазона изменения числа Кнудсена. Реализация алгоритма данного метода произведена с применением системы компьютерной алгебры Maple 17. Результаты вычислений представлены в таблице.

\section{Анализ результатов}

Анализ данных, представленных в таблице, показал, что приведенный поток массы зависит от коэффициента аккомодации тангенциального импульса, причем наибольшее отклонение значений наблюдается при приближении к свободномолекулярному режиму. Уменьшение коэффициента аккомодации тангенциального импульса приводит к увеличению массового потока через поперечное сечение канала.

Для режима течения, близкого к свободномолекулярному $(K n \gg 1)$, выражения (18) и (19) для приведенных потоков тепла и массы могут быть представлены в виде рядов по малому параметру $K n^{-1}$. В этом случае, ограничиваясь членами разложения прядка $K n^{-2}$, получим

$$
\begin{gathered}
J_{Q}=-\frac{6(2-\alpha)}{\alpha \sqrt{\pi}}-\frac{2 \sqrt{\pi}\left(\alpha^{2}+6(1-\alpha)\right)}{5 \alpha^{2}} \frac{1}{K n} \ln \left(\frac{1}{K n}\right), \\
J_{M}=-\frac{2}{9} J_{Q} .
\end{gathered}
$$

Линейные члены относительно $K n^{-1}$ в (21) определяют приведенные потоки тепла и массы через поперечное сечение цилиндрического канала под действием

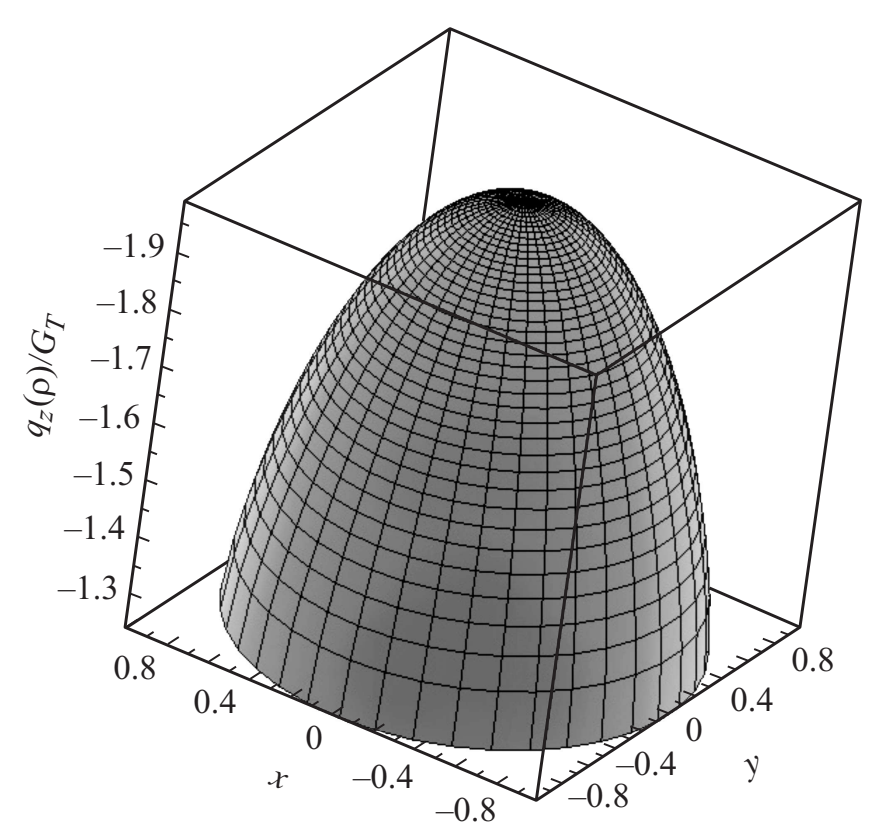

Рис. 1. Профиль вектора потока тепла в канале $q_{z}(\rho) / G_{T}$ при $\alpha=1, K n=10^{3}$.

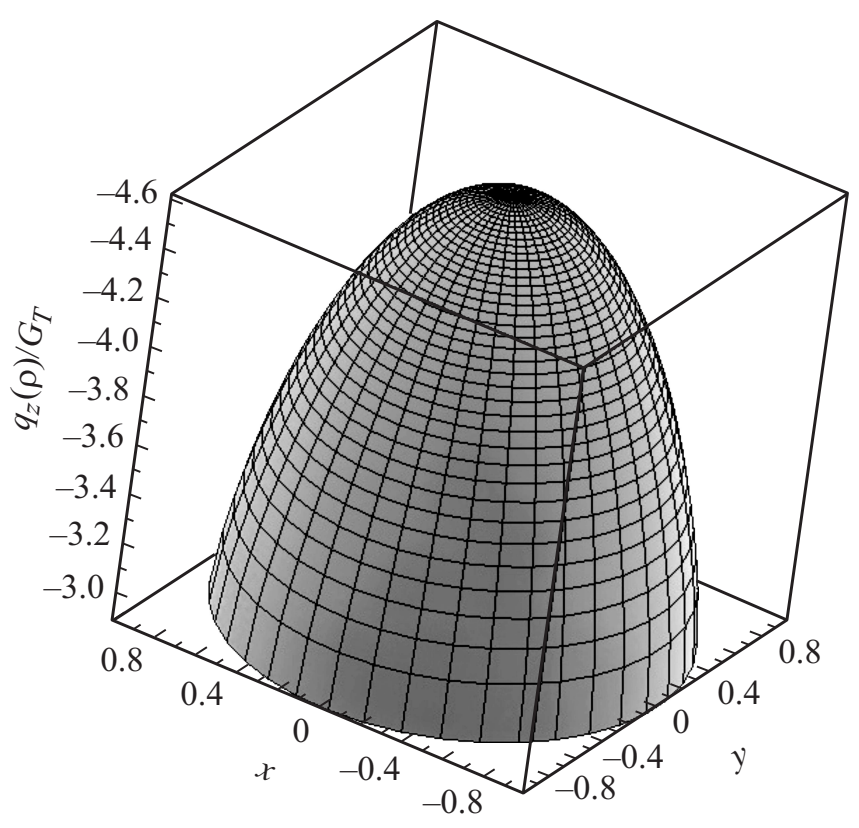

Рис. 2. Профиль вектора потока тепла в канале $q_{z}(\rho) / G_{T}$ при $\alpha=0.6, K n=10^{3}$.

постоянного градиента температуры в свободномолекулярном режиме и совпадают при $\alpha=1$ с выражениями $J_{Q}=-6 / \sqrt{\pi}$ и $J_{M}=4 /(3 \sqrt{\pi})$ из работы [6] при использовании диффузной модели отражения молекул газа от поверхности.

Для режимов течения, близких к гидродинамическому, анализ выражений (18) и (19) приводит к следую- 


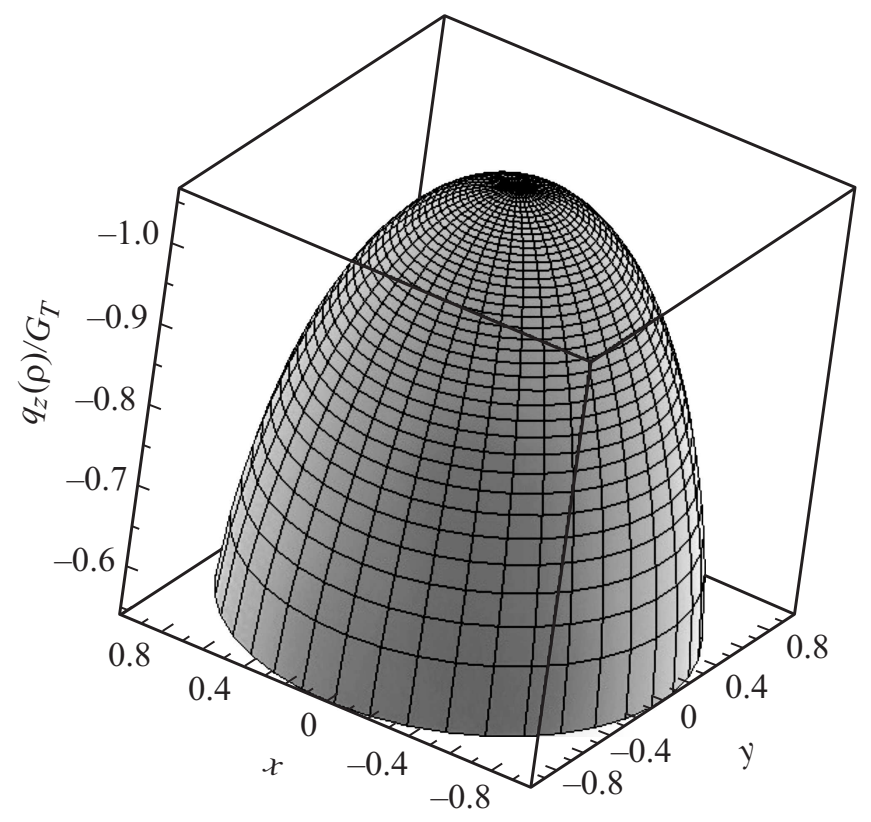

Рис. 3. Профиль вектора потока тепла в канале $q_{z}(\rho) / G_{T}$ при $\alpha=1, K n=1$.

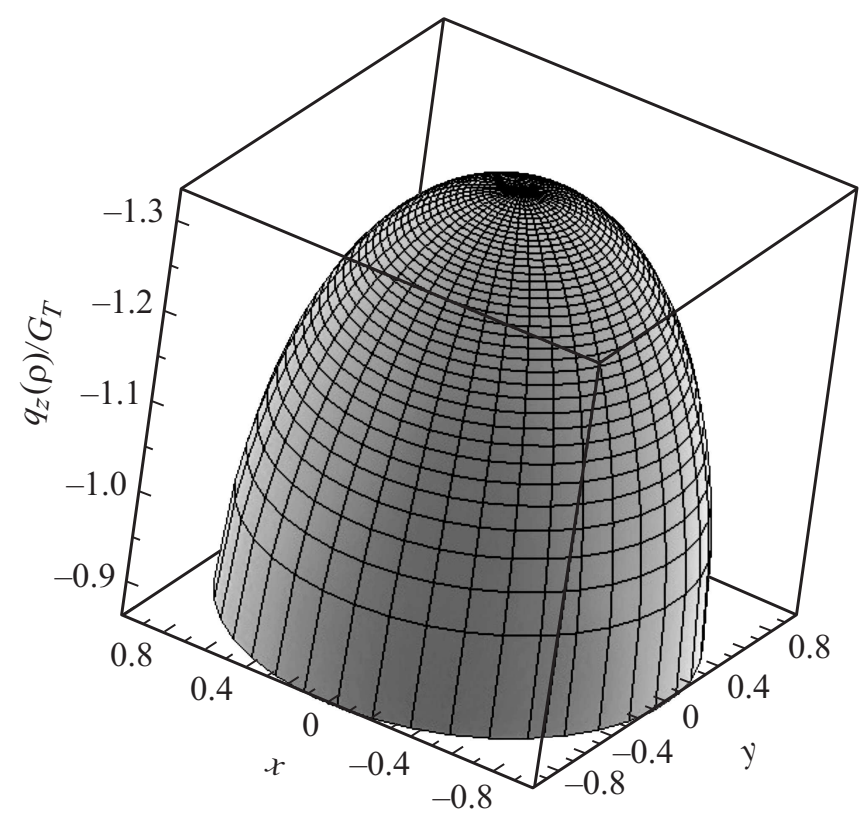

Рис. 4. Профиль вектора потока тепла в канале $q_{z}(\rho) / G_{T}$ при $\alpha=0.6, K n=1$.

щим результатам:

$$
J_{Q}=-\frac{15 G_{T} K n}{4}, \quad J_{M}=\frac{5 G_{T} K n}{6} .
$$

Таким образом, для режимов, близких к гидродинамическому режиму, приведенные потоки тепла и массы не зависят от коэффициента аккомодации тангенциального импульса. Последнее утверждение подтверждается результатами, приведенными в таблице для $K n<0.0010$, а выражение для $J_{Q} / G_{T}$ в (22) совпадает с приведенным в работе [17]. Из выражения (22) для $J_{M} / G_{T}$ вытекает, что коэффициент теплового скольжения равен $5 / 6$, что совпадает со значением, найденным в [22]. Как следует из таблицы, полученные в настоящей работе результаты отличаются от аналогичных результатов, полученных в работах $[6,14]$ в рамках S-модели кинетического уравнения Больцмана. Отличие обусловлено тем фактом, что значения макропараметров газа в канале существенно зависят от выбора модели интеграла столкновений [6].

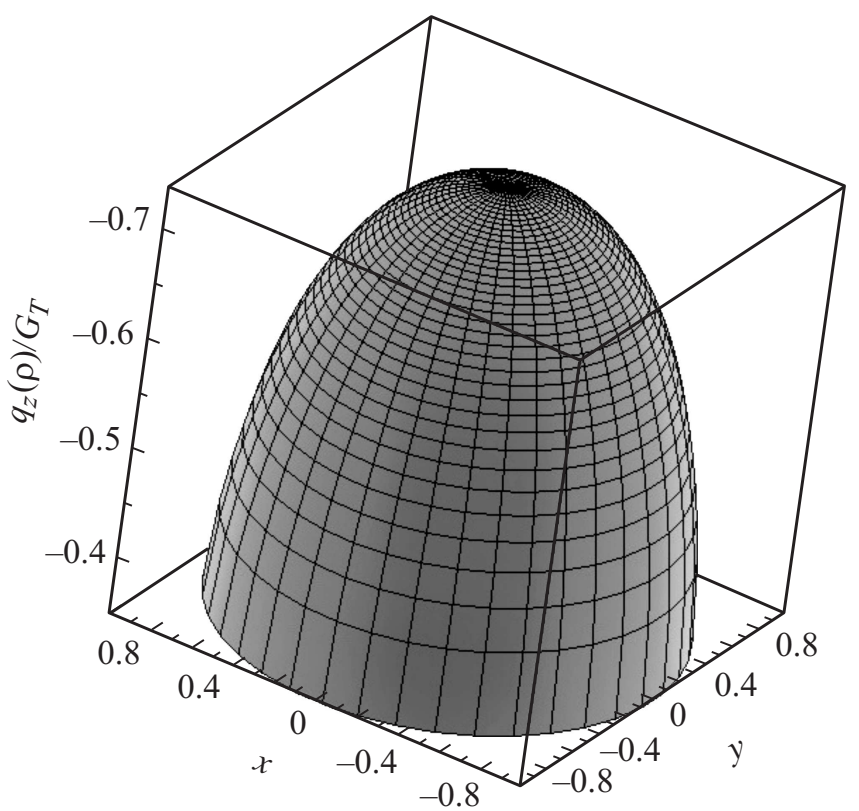

Рис. 5. Профиль вектора потока тепла в канале $q_{z}(\rho) / G_{T}$ при $\alpha=1, K n=0.5$.

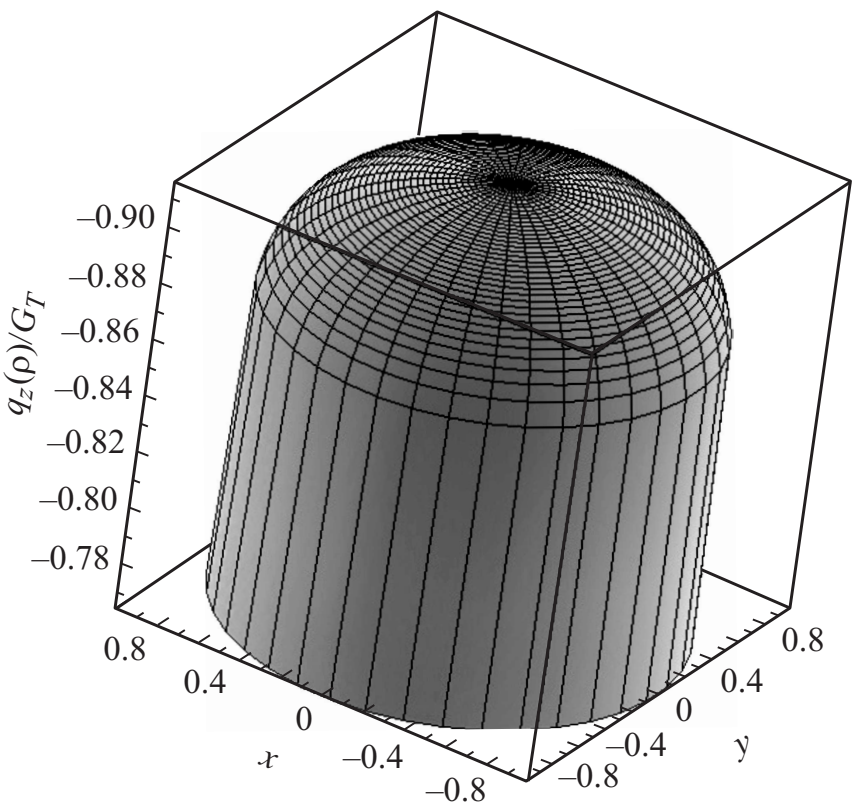

Рис. 6. Профиль вектора потока тепла в канале $q_{z}(\rho) / G_{T}$ при $\alpha=0.1, K n=0.5$. 
Профили распределения z-компоненты вектора потока тепла по сечению канала, рассчитанные согласно (17) в зависимости от коэффициента аккомодации тангенциального импульса молекул газа, показаны на рис. 1-6. Рис. 1,3,5 описывают процессы теплопереноса через поперечное сечение канала в случае полной аккомодации молекул газа на поверхности. Из представленных рисунков видно, что распределение компоненты вектора потока тепла имеет минимум в начале координат. При $K n \gg 1$ и $\alpha=1$ профиль вектора потока тепла представляет собой параболоид вращения с осью, совпадающей с осью цилиндра. При $K n \ll 1$ профиль вектора потока тепла образует плоскость, перпендикулярную оси цилиндра, отклонение от которой имеет место в тонком пристеночном слое (слое Кнудсена), что совпадает с выводами работы [17].

\section{Заключение}

В рамках кинетического подхода найдено решение задачи о переносе тепла и массы газа в цилиндрическом канале под действием постоянного градиента температуры с использованием диффузно-зеркальной модели отражения Максвелла. Построены профили вектора потока тепла в зависимости от коэффициента аккомодации тангенциального импульса, вычислены значения потока тепла через поперечное сечение канала в широком диапазоне изменения числа Кнудсена. Проведено сравнение с аналогичными результатами при полной аккомодации молекул на стенках канала.

Работа выполнена при поддержке гранта РФФИ по научному проекту № 16-29-15116 офи_м.

\section{Список литературы}

[1] Ухов А.И., Борисов С.Ф., Породнов Б.Т. // Теплофизика и аэромеханика. 2010. Т. 17. № 1. С. 141-150.

[2] Коган М.Н. Динамика разреженного газа. Кинетическая теория. М.: Наука, 1967. 440 с.

[3] Cercignani C., Lampis M. // Transport Theory Statist. Phys. 1971. Vol. 1. P. 101-114.

[4] Гулакова С.В., Попов В.Н. // ЖТФ. 2015. Т. 85. № 4. С. 1-6.

[5] Siewert C.E. // Z. Angew. Math. Phys. 2003. Vol. 54. P. 273 203.

[6] Шарипов Ф.М., Селезнев В.Д. Движение разреженных газов в каналах и микроканалах. Екатеринбург: УрО РАН, 2008. $230 \mathrm{c}$.

[7] Lo S.S., Loyalka S.K., Storvick T.S. // J. Chem. Phys. 1984. Vol. 81. P. 2439-2949.

[8] Ewart T., Graur I., Perrier P., Meolans J.G. // Microfluidics and Nanofluidics. 2007. Vol. 26. N 6. P. 689-695

[9] Silva E., Rojas-Cardenas M., Deschamps C.J. // International J. Refrigeration. 2016. Vol. 66. P. 121-132.

[10] Sharipov F.M. // J. Vac. Sci. Technol. A. 1999. Vol. 17. N 5. P. 3062-3066.

[11] Titarev V.A., Shakhov E.M. // Comput. Math. Math. Phys. 2010. Vol. 50. N 7. P. 1221-1237.
[12] Гермидер О.В., Попов В.Н., Юшканов А.А. // ЖТФ. 2016. T. 86. № 6. C. 37-41.

[13] Naris S., Valougeorgis D. // European J. Mechanics B / Fluids. 2008. Vol. 27. P. 810-822.

[14] Siewert C.E., Valougeorgis D. // J. Quantitative Spectroscopy \& Radiative Transfer. 2002. Vol. 72. P. 531-550.

[15] Taheri P., Bahrami M. // Phys. Rev. 2012. Vol. 86. P. 1-9.

[16] Kamphorst C.H., Rodrigues P., Barichello L.B. // Appl. Mathematics. 2014. Vol. 5. P. 1516-1527.

[17] Germider O.V., Popov V.N., Yushkanov A.A. // J. Eng. Phys. Thermophysics. 2016. Vol. 89. N 5. P. 1338-1343.

[18] Graur I., Sharipov F. // European J. Mechanics B / Fluids. 2008. Vol. 27. P. 335-345.

[19] Pantazis S., Varoutis S., Hauer V., Day C, Valougeorgis D. // Vacuum. 2011. Vol. 85. P. 1161-1164.

[20] Cercignani C. Mathematical Methods in Kinetic Theory. NY: Plenum Press, 1969. 227 p.

[21] Курант Р. Уравнения с частными производными. М.: Мир, 1964. $830 \mathrm{c}$.

[22] Латышев А.В., Юшканов А.А. Кинетические уравнения типа Вильямса и их точные решения. М.: МГОУ, 2004. $271 \mathrm{c}$. 